\title{
TempliPhi, $\phi 29$ DNA Polymerase Based Rolling Circle Amplification of Templates for DNA Sequencing
}

John R. Nelson, Yuyang Christine Cai, Theresa L. Giesler, Joseph W. Farchaus, Shanmuuga T. Sundaram, Maria Ortiz-Rivera, Lou P. Hosta, Peter L. Hewitt, J. Anthony Mamone, C. Palaniappan, and Carl W. Fuller Genomics Reagents Department, Amersham Biosciences, Piscataway, NJ, USA

BioTechniques 32:S44-S47 (June 2002)

\section{ABSTRACT}

We have developed a novel, isothermal DNA amplification strategy that employs $\phi 29$ DNA polymerase and rolling circle amplification to generate high-quality templates for DNA sequencing reactions. The TempliPhi ${ }^{T M}$ DNA amplification kits take advantage of the fact that cloned DNA is typically obtained in circular vectors, which are readily replicated in vitro using $\$ 29$ DNA polymerase by a rolling circle mechanism. This single subunit, proofreading DNA polymerase has excellent processivity and strand displacement properties for generation of multiple, tandem double-stranded copies of the circular DNA, generating as much as 107-fold amplification. Large amounts of product $(1-3 \mu \mathrm{g})$ can be obtained in as little as 4 hours. Input DNA can be as little as $0.01 \mathrm{ng}$ of purified plasmid DNA, a single bacterial colony, or a $1 \mu \mathrm{L}$ of a saturated overnight culture. Additionally, the presence of an associated proofreading function within the $\phi 29$ DNA polymerase ensures high-fidelity amplification. Once completed, the product DNA can be used directly in sequencing reactions. Additionally, the properties of $\phi 29$ DNA polymerase and its use in applications such as amplification of human genomic DNA for genotyping studies is discussed.

\section{INTRODUCTION}

The TempliPhi ${ }^{\mathrm{TM}}$ DNA Sequencing Template Amplification Kit is a novel product that has been developed specifically to prepare templates for DNA sequencing. The TempliPhi method utilizes bacteriophage $\$ 29$ DNA polymerase enzyme (1) to exponentially amplify circular DNA templates by rolling circle amplification (RCA) (7). Multiple strand displacement amplification of any DNA, including linear and genomic DNAs, can also be performed efficiently using this polymerase. A proofreading enzyme, it has both high processivity and potent strand displacement activities. Together, these properties allow us to use $\phi 29$ DNA polymerase to achieve isothermal DNA amplification with random-sequence hexamer primers. We have previously shown that phosphorothioate modification of the primer prevents the DNA polymerase from degrading the primers and dramatically stimulates reaction kinetics (3). This isothermal amplification method is able to produce microgram quantities of DNA from picograms of starting material in a few hours. This method provides great potential for general amplification of DNA samples.

\section{MATERIALS AND METHODS}

Determination of the Fidelity of $\$ 29$ DNA Polymerase

The fidelity of $\phi 29$ polymerase was measured by the screening method described by Kunkel (6). Reactions (50 $\mu$ L total volume) containing 1 ng of pBluescript $^{\circledR}$ II SK(+) (Stratagene, La Jolla, CA, USA) and 200 pmol of random hexamer (Amersham Biosciences, Piscataway, NJ, USA) were heated to $95^{\circ} \mathrm{C}$ for 3 minutes. These were then chilled, $200 \mu \mathrm{M}$ dNTPs and $10 \mathrm{U}$ of $\$ 29$ DNA polymerase (Amersham Biosciences) were added, and reactions were incubated at $30^{\circ} \mathrm{C}$ for 16 hours. DNA samples were digested with SacI (New England Biolabs, Beverly, MA, USA), heat inactivated, ligated, transformed into XL-1 Blue supercompetent cells (Stratagene), and plated onto LB/amp plates containing X-Gal and isopropyl $\beta$-D-thiogalactosude (IPTG). Plates were incubated at $37^{\circ} \mathrm{C}$ for 16 hours and then the blue and white colonies were counted. The error rate was calculated using the equation $\mathrm{ER}=\mathrm{mf}$ I (bpXd), where $\mathrm{mf}$ is the mutation frequency, bp is the number of detectable sites (350) in the lac $Z$ gene fragment of the vector, and $\mathrm{d}$ is the number of template doublings (13.3 for 10000 -fold amplification). We counted 1488 blue colonies and 21 white colonies. Data are the average of two independent experiments.

\section{TempliPhi 100 Reactions}

The TempliPhi 100 DNA Sequencing Template Amplification kit (Amersham Biosciences) was used as described in the manufacturer's protocol booklet with the following notable exceptions. The template ( $1 \mathrm{ng}$ vector DNA, 1 colony or $1 \mu \mathrm{L}$ of saturated overnight culture) was added to $5 \mu \mathrm{L}$ of sample buffer. During the denaturation step, a reaction premix of $5 \mu \mathrm{L}$ reaction buffer +0.2 $\mu \mathrm{L}$ enzyme mix for each sample was prepared. Once tubes were cooled from denaturation temperature, $5 \mu \mathrm{L}$ of this freshly made premix was added to each sample and the resulting reaction mixtures $(10 \mu \mathrm{L})$ were incubated at $30^{\circ} \mathrm{C}$ for the indicated times.

\section{DNA Quantification}

TempliPhi products were diluted 10-fold with Tris EDTA (TE) and vortex mixed. Afterwards, samples were centrifuged 
briefly, mixed a second time by pipet, and 10\% (10-20 $\mu \mathrm{L})$ of the diluted TempliPhi product was added to $80-90 \mu \mathrm{L}$ of TE in each well of Microfluor ${ }^{\circledR} 2$ white flat bottom 96-well plates (ThermoLabsystems, Franklin, MA, USA). Picogreen ${ }^{\circledR}$ dsDNA Quantitation Reagent (Molecular Probes, Eugene, OR, USA) was diluted 1:25 in TE, and $100 \mu \mathrm{L}$ of this was added to each sample. Lambda DNA supplied with the Picogreen dsDNA Quantitation Kit was used as the concentration standard. Samples were read on the HTS 7000 Plus Bio Assay reader (Perkin Elmer, Wellesley, MA, USA). Background subtracted RFU data were used to calculate standard curves and yields. Data are the average of at least triplicate samples for each time point.

\section{DNA Sequencing}

Individual isolates (96 random colonies) from a library of $2-4 \mathrm{~kb}$ inserts in pUC18 were hand picked and amplified for 8 hours using the TempliPhi 100 kit. The amplified material was diluted 1:10 in water and $10 \mu \mathrm{L}$ was used for sequencing. Each sample was sequenced with both the -40 and the -28 primer and with both the DYEnamic ${ }^{\mathrm{TM}}$ ET terminator kit (Amersham Biosciences; 605 PHRED 20 nt average) or the BigDye ${ }^{\mathrm{TM}} \mathrm{v} 3$ terminator kit (ABI, Foster City, CA, USA; 584 PHRED $20 \mathrm{nt}$ average) and run on an ABI model 3700 sequencer.

\section{RESULTS AND DISCUSSION}

\section{Processivity and Strand Displacement}

$\phi 29$ DNA polymerase is a highly processive enzyme that incorporates at least 70000 nucleotides per binding event (1). In a processivity assay using M13 single-stranded DNA as a template and a limiting amount of $\phi 29$ DNA polymerase, replication of the circle proceeds by rolling circle replication (Figure 1). $\$ 29$ DNA polymerase does not apparently pause when encountering double-stranded template, performing strand displacement synthesis in the absence of added helicase or SSB. High molecular weight products are observed after 9 hours and are difficult to size as they fail to migrate into the gel. Fragments of intermediate size are not observed, indicating the product has been synthesized during a single binding event. $\$ 29$ DNA polymerase incorporates nucleotides at $25-50 \mathrm{nt} /$ second, which would suggest that the potential size of this product is over $800000 \mathrm{nt}$ long. We have observed linear kinetics for 2 days using this enzyme (data not shown).

\section{Fidelity of $\phi 29$ DNA Polymerase}

This polymerase has an associated $3^{\prime}-5^{\prime}$ exonuclease "proofreading" activity (2) and has a reported error rate of $5 \times 10^{-6}(5)$, about 100 -fold lower than that of Taq DNA polymerase (4). Results using a TempliPhi method of amplification confirmed this published error rate for $\phi 29$ DNA polymerase. Following a modified Kunkel method (6), we found an error rate of $3 \times 10^{-6}$. In addition, sequencing experiments indicate that $\phi 29$ DNA polymerase faithfully replicates input DNA. In the DNA sequence data from primer walks using TempliPhi amplified M13mp18 and pUC18 DNA (10 000-fold amplified, approxi- mately 20000 nt of sequence) using 24 forward and 24 reverse vector specific primers in a total of eleven 96 -well plate sequencing runs on MegaBACE ${ }^{\mathrm{TM}} 1000$ we have detected no amplification errors (data not shown).

\section{Amplification Kinetics}

TempliPhi amplification is a very powerful process that requires little starting material. Typically there is a brief lag followed by rapid synthesis of product until the supply of nucleotides is exhausted (Figure 2). Although the standard TempliPhi protocol uses $1 \mathrm{ng}$ of purified DNA as the input template for optimal kinetics, we have found that as little as 0.1 picogram of pUC18 DNA can be amplified efficiently (Figure $3 \mathrm{~A})$. As long as the specific target template is $>0.01$ picogram, the target will out-compete nonspecific amplification of the random hexamers. The result of nonspecific amplification can be seen in lanes containing no input DNA (Figure 3, A and B). We have cloned and sequenced this nonspecific amplified material from reactions containing no input template DNA, and with the exception of a few clones with homology to Escherichia coli and our expression vector, the majority of the clones have no significant homology to any DNA in the entire National Center for Biotechnology Information (NCBI) database.

\section{Amplification of Plasmids Directly from Cells}

Plasmid DNA present in bacterial colonies can be used directly as template for TempliPhi amplifications. This is a great convenience since clones can be screened at the colony stage

\section{Time (hrs)}
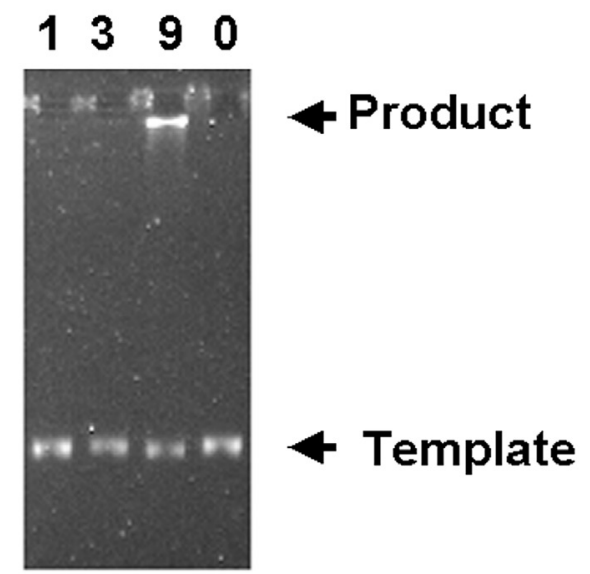

Figure 1. Strand displacement and processivity assay. The strand displacement and processivity activities of $\phi 29$ DNA polymerase were demonstrated using a single primed M13 (single-stranded circle) extension assay. Reactions containing $25 \mathrm{ng}$ of M13mp18 single-stranded DNA and 5 pmol of -40 universal primer in $10 \mu \mathrm{L} \mathrm{TE}$ were heated to $95^{\circ}$ for 3 minutes and slow cooled to room temperature. These were then chilled, TempliPhi reaction buffer and $0.5 \mathrm{U}$ (an amount known to be subsaturating) of $\$ 29$ DNA polymerase (Amersham Biosciences) were added, and reactions were incubated at $300 \mathrm{C}$ for the indicated times. The DNA products were then resolved on a $0.6 \%$ agarose electrophoresis gel $1 \times$ Tris-buffered EDTA. 
without having to wait for liquid cultures to grow and without the need for DNA plasmid preparation. As little as one part in 10000 of a single colony can act as a good template forTempliPhi amplification, generating more than $1 \mathrm{mg}$ of product in 4-6 hours (data not shown). However, amplification is more consistent when one part in 1000 or more is used, up to the content of a full colony. Although this data was obtained using the high copy number plasmid pUC18, we also tested amplification of lower copy number plasmids including pBR322, pBeloBAC11, and pBACe3.6. In general, for these low copy plasmids, $1 \%$ to $100 \%$ of the contents of a colony should be used for amplifications performed for $8-16$ hours at $30^{\circ} \mathrm{C}$ to achieve maximal yields.

We have observed that although chromosomal DNA can be efficiently amplified using the TempliPhi method, there is no evidence for the amplification of the host DNA after amplification of whole cultures containing plasmid DNA (Figure 3B, lane 1). We believe this is the result of using a denaturation step $\left(95^{\circ} \mathrm{C}\right.$ for 3 minutes) that is harsh enough to release plasmid DNA but not to lyse the bacterial cells.

M13 plaques are also excellent starting material for amplification. Material from M13 plaques picked directly from YT agar plates were serially diluted in sample buffer and amplified. We obtained an average of greater than $500 \mathrm{bp}$ of sequence data with at least $98 \%$ accuracy using $1 / 100^{\text {th }}$ of an M13 plaque as amplification starting template (data not shown). Supernates from M13 phage growth in bacterial culture may also be used. We have used between 0.02 and $1 \mu \mathrm{L}$ of M13 phage containing bacterial culture supernatants in 4 hour TempliPhi reactions to successfully generate DNA sequencing templates (data not shown). Interestingly, since the products of the amplification of M13 single-stranded DNA circles are double stranded, both strands of M13 clones can be sequenced.

Liquid cultures also provide good templates for TempliPhi reactions. This is particularly useful to researchers who prefer to grow small overnight cultures for archival storage. There is no significant difference in amplification yield when using

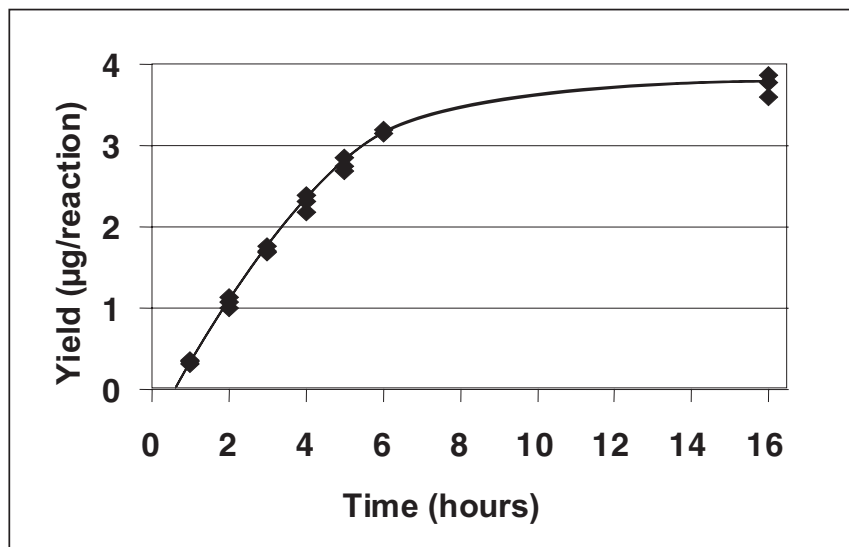

Figure 2. TempliPhi DNA amplification kinetics. The 10000 reaction TempliPhi DNA Sequencing Template Amplification Kit was used as follows: supercoiled pUC18 DNA (1 ng) was added to $10 \mu \mathrm{L}$ of sample buffer, heated at $95^{\circ} \mathrm{C}$ for $3 \mathrm{~min}$ to denature the template, and chilled to $4^{\circ} \mathrm{C}$. To this, $10 \mu \mathrm{L}$ of TempliPhi Premix from the kit was added, and tubes were placed at $30^{\circ} \mathrm{C}$ for the indicated times. To stop the amplification reaction, samples were heated to $65^{\circ} \mathrm{C}$ for $10 \mathrm{~min}$ to inactivate the $\phi 29$ DNA polymerase and then quantified.
$0.001-1.0 \mu \mathrm{L}$ of a saturated overnight $E$. coli culture as initial template for TempliPhi amplifications (Figure 3B). We have obtained good sequencing data by amplification of a culture dilution that contains between 1 and 10 E. coli cells (DNA from the sixth lane of Figure 3B). For low copy number plasmids, as little as $0.03 \mu \mathrm{L}$ of overnight shaking culture is sufficient template for amplification (data not shown). Again, as described for colonies, longer incubations at $30^{\circ} \mathrm{C}$ for these types of vectors is beneficial. Even a small portion of frozen glycerol stock can be amplified directly in TempliPhi reactions.

\section{Inhibitors of Amplification}

We have found that large amounts of bacterial cells can inhibit amplification reaction kinetics. In most cases a dilution of bac-

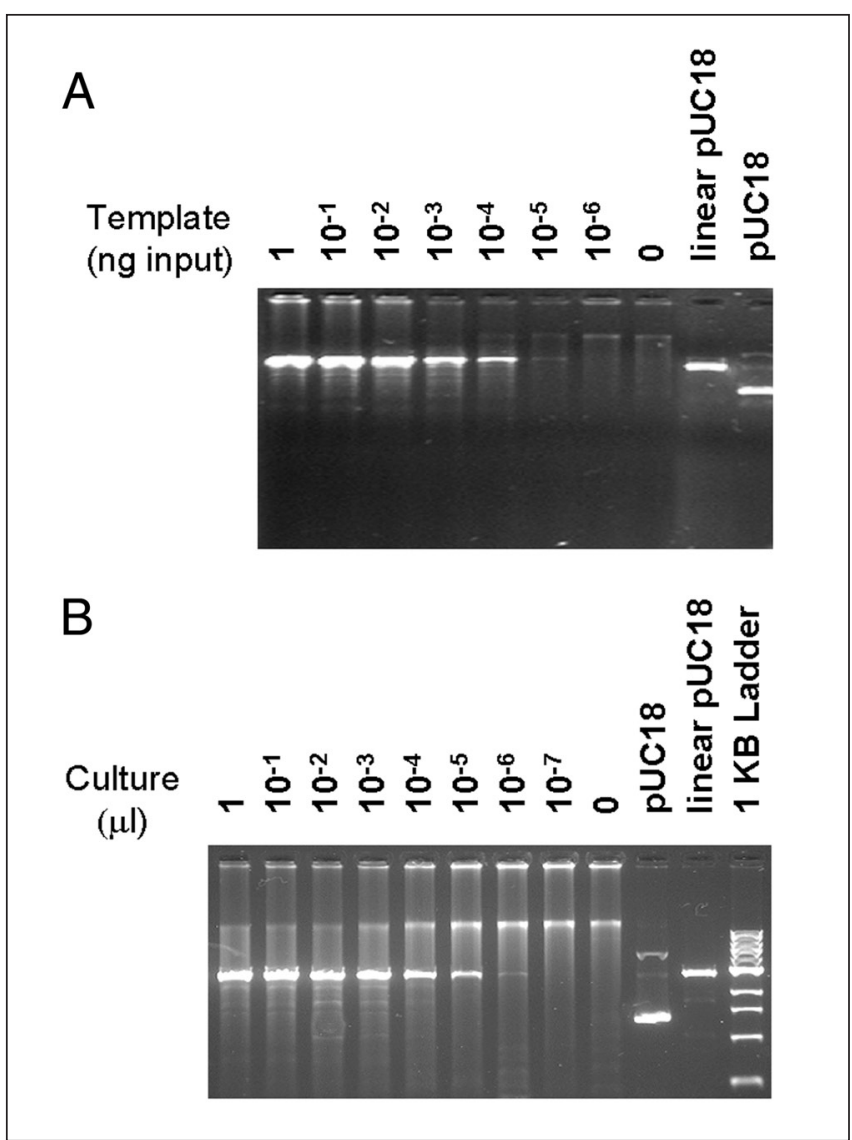

Figure 3. Template titration assays. TempliPhi amplification reactions were performed at $30^{\circ} \mathrm{C}$ for 4 hours. (A) Purified, supercoiled pUC18 DNA was 10 -fold serially diluted in sample buffer to contain $1 \mathrm{ng}$ down to $10^{-6} \mathrm{ng}$ ( 1 attogram) of DNA and used as template for amplification. (B) A $37^{\circ} \mathrm{C}$ standing overnight LB culture of $E$. coli strain DH5 $\alpha$ (approximately $3.5 \times 10^{8}$ cells $/ \mathrm{mL}$ ) containing pUC18 vector was serially diluted in sample buffer used as template for amplification. After amplification, the reaction mixture was heat inactivated at $65^{\circ} \mathrm{C}$ for 10 minutes and a portion of the DNA product was digested with EcoRI for 3 hours at $37^{\circ} \mathrm{C}$. Restriction digest products $(15 \%$ of original amplification reaction product) were resolved on $0.6 \%$ agarose gels in $0.5 \times$ Trisbuffered EDTA. The $1 \mathrm{~kb}$ ladder (New England Biolabs) was used as the molecular weight standard. Gels were stained with GelStar ${ }^{\circledR}$ Nucleic Acid Gel Stain (BioWhittaker Molecular Applications, Rockland, ME, USA) using manufacturer's recommendations. Gels were visualized with the Typhoon 8600 Variable Mode Imager (Amersham Biosciences). 
terial colonies or careful attention to the amount of colony transferred into the TempliPhi reaction improves amplification kinetics and overall yields. While growth media has essentially no affect on the reaction, we observe up to a 6-fold decrease in amplification kinetics when using cultures grown to high cell density $\left(\mathrm{OD}_{600}>3\right)$ or aerated cultures grown in rich media such as Terrific Broth and $2 \times$ YT (data not shown). We have obtained excellent results using one microliter from standing (not shaking) overnight cultures in LB as input for the TempliPhi reaction.

\section{DNA Sequencing}

The TempliPhi reaction products do not need to be processed prior to DNA sequencing. Since the amplification depletes the nucleotides, and the random hexamers will not anneal under typical DNA sequencing conditions, samples can be sequenced directly after amplification using any of the commercially available dye terminator fluorescent DNA sequencing kits. Interestingly, sequenced samples that are processed after the sequencing reaction by G-50 spin columns or precipitation on carboxylate-modified magnetic beads typically have a significant portion of the template DNA selectively removed (data not shown). This may lead to improved results on capillary sequencing instruments that are known to be less tolerant to high concentrations of template DNA than slab gel instruments (for protocols using carboxylate modified magnetic beads, see the DOE Joint Genome Institute [JGI] Web site: www.jgi.doe.gov).

An additional benefit of the TempliPhi method relates to the observation that amplification continues until the supply of nucleoside triphosphates is exhausted. As a result, every amplification finishes with the same concentration of DNA product. This effectively normalizes all of the DNA samples for sequencing. This consistency can improve DNA sequencing success rate, which is strongly influenced by variability in DNA concentration.

We have achieved excellent success rates using the TempliPhi method to generate sequencing templates for all of the major DNA sequencing instruments. Using manual procedures, success rates on the ABI model 3700 and 3100 capillary sequencers using either BigDye or DYEnamic ET terminator sequencing kits are typically over $85 \%$, with excellent read lengths. The JGI currently uses TempliPhi amplification with their automated production sequencing facility and MegaBACE sequencing instruments, and in March of 2002 sequenced a total of 923 million Q20 bases in 8621 runs with a $91.37 \%$ success rate and read length of $599 \mathrm{nt}$ (P. Richardson, personal communication).

\section{Amplification of Linear DNA}

Using materials similar to those found in the TempliPhi kit, we have recently demonstrated very efficient amplification of 50 $\mathrm{kb}$ bacteriophage lambda DNA, despite the fact that this DNA is linear and not circular. Also, using $1 \mathrm{ng}$ of human genomic DNA we have generated over $20 \mu \mathrm{g}$ of high molecular weight DNA, which is representative of the input sample and maintains linkage information over $20000 \mathrm{nt}$ in length (manuscript submitted). We anticipate that the DNA contained in a single human cell will be amplified reliably by this new method as well. Using both quantitative PCR and hybridization analysis, we have shown this method to give equivalent amplification from numerous locations throughout the genome. Many genetic analysis methods, including most SNP testing methods, utilize PCR to amplify specific DNA regions prior to the actual genetic test. We have determined that chromosomal DNA amplified by this new method can be used very effectively as PCR templates. Feasibility experiments are under way to test this method using a variety of sources of input template DNA, and to confirm that the product of this method can be used in place of chromosomal DNA. We hope to develop a kit using $\$ 29$ DNA polymerase for efficient amplification of linear DNA templates in the near future.

\section{REFERENCES}

1.Blanco, L., A. Bernad , J.M. Lazaro, G. Martin, C. Garmendia, and M. Salas. 1989. Highly efficient DNA synthesis by the phage phi 29 DNA polymerase. Symmetrical mode of DNA replication. J. Biol. Chem. 264:89358940.

2.Blanco, L. and M. Salas. 1996. Relating structure to function in phi29 DNA polymerase. J. Biol. Chem. 271:8509-8512.

3.Dean, F.B., J.R. Nelson, T.L. Giesler, and R.S. Lasken. 2001.Rapid amplification of plasmid and phage DNA using phi29 DNA polymerase and multiply-primed rolling circle amplification. Genome Res. 11:1095-1099.

4.Dunning, A.M., P. Talmud, and S.E. Humphries. 1988. Errors in the polymerase chain reaction. Nucleic Acids Res. 16:10393.

5.Esteban, J.A., M. Salas, and L. Blanco. 1993. Fidelity of phi 29 DNA polymerase. Comparison between protein-primed initiation and DNA polymerization. J. Biol. Chem. 268:2719-2726.

6.Kunkel, T.A. 1985. The mutational specificity of DNA polymerase-beta during in vitro DNA synthesis. Production of frameshift, base substitution, and deletion mutations. J. Biol. Chem. 260:5787-5796.

7.Lizardi, P.M., X. Huang, Z. Zhu, P. Bray-Ward, D.C.Thomas, and D.C. Ward. 1998. Mutation detection and single-molecule counting using isothermal rolling-circle amplification. Nat Genet. 19:225-232.

\section{Address correspondence to:}

Dr. C. Palaniappan

Genomics Reagents Department

Amersham Biosciences

800 Centential Avenue

Piscataway, NJ 08855, USA

e-mail:palani@am.amershambiosciences.com 